\title{
The Sirt1 activator, SRT1720, attenuates renal fibrosis by inhibiting CTGF and oxidative stress
}

\author{
YUNZHUO REN ${ }^{1,2^{*}}$, CHUNYANG DU ${ }^{1,2^{*}}$, YONGHONG SHI ${ }^{1,2}$, \\ JINGYING WEI ${ }^{1,2}$, HAIJIANG WU ${ }^{1,2}$ and HUIXIAN CUI ${ }^{3}$ \\ ${ }^{1}$ Department of Pathology, Hebei Medical University; ${ }^{2}$ Hebei Key Laboratory of Kidney Diseases; \\ ${ }^{3}$ Department of Anatomy, Hebei Medical University, Shijiazhuang, Hebei 050017, P.R. China
}

Received December 17, 2015; Accepted March 15, 2017

DOI: $10.3892 /$ ijmm.2017.2931

\begin{abstract}
The transforming growth factor- $\beta 1$ (TGF- $\beta 1$ )/ connective tissue growth factor (CTGF) pathway plays an important role in the pathogenesis and progression of chronic kidney disease. Oxidative stress is also involved in TGF- $\beta 1$ signalling. Sirtuin 1 (Sirt1) exerts a number of pleiotropic effects, protecting against renal disease, including inhibiting fibrosis and oxidative metabolism. In this study, we investigated the role of the Sirt1 activator, SRT1720, in unilateral ureteral obstruction (UUO)-induced tubulointerstitial fibrosis and aimed to determine whether this role depends on the inhibition of oxidative stress and the TGF- $\beta 1 / C T G F$ pathway. Renal fibrosis was induced by UUO in CD1 mice. SRT1720 (100 mg/ $\mathrm{kg}$ ) was administered by intraperitoneal injection for 3 days prior to UUO and this was continued for 7 days following UUO. Histological changes were examined by Masson's trichrome staining. The expression of fibrosis-related factors was evaluated by immunohistochemistry, western blot analysis and RT-qPCR. Apoptosis was also examined. We also examined the superoxide dismutase (SOD), malondialdehyde (MDA), glutathione peroxidase (GPx) and reduced glutathione (GSH) levels. UUO induced renal fibrosis and apoptosis and decreased Sirt1 expression. The administration of SRT1720 increased the Sirtl levels and partially attenuated UUO-induced renal fibrosis and apoptosis. Furthermore, SRT1720 attenuated the levels of oxidative stress (it decreased the MDA levels, and increased the SOD, GPx and GSH levels), which suggests that it protected the cells against ROS-induced damage. Moreover, SRT1720 effectively inhibited the levels of TGF- $\beta 1 /$ CTGF induced by UUO.
\end{abstract}

Correspondence to: Dr Huixian Cui, Department of Anatomy, Hebei Medical University, 361 East Zhongshan Road, Shijiazhuang, Hebei 050017, P.R. China

E-mail: cuihxf@126.com

*Contributed equally

Key words: sirtuin 1, apoptosis, fibrosis, oxidative stress, transforming growth factor- $\beta 1 /$ connective tissue growth factor
On the whole, these findings indicate that the Sirt1 activator, SRT1720, exerts protective effects against UUO-induced tubulointerstitial fibrosis. The mechanisms of action of SRT1720 may include, at least in part, the suppression of renal oxidative stress and the TGF- $\beta 1 /$ CTGF signalling pathway. The Sirt1 activator may therefore be prove to be a potent therapeutic agent for the treatment of fibrotic kidney disease.

\section{Introduction}

Renal disease can advance to tubulointerstitial fibrosis and has been proposed as a final common pathway to end-stage renal disease. Uunilateral ureteral obstruction (UUO) results in a number of pathophysiological and morphological changes in the renal parenchyma, including interstitial fibrosis, inflammation and apoptotic changes in tubular and interstitial cells (1). Among the mechanisms responsible for renal fibrogenesis, the transforming growth factor- $\beta$ (TGF- $\beta$ ) signalling pathway is known to play a pivotal role in renal tubulointerstitial fibrosis. Through the autocrine and paracrine stimulation of cells by TGF- $\beta 1$, connective tissue growth factor (CTGF) is released and synthesised, which plays a role in fibrogenesis (2).

Reactive oxygen species (ROS) are also believed to play a key role in fibrosis. The renal tubulointerstitium requires high oxygen consumption for active solute transport and reabsorption, and the tubulointerstitium is susceptible to oxidative stress. Increased ROS accumulation leads to renal fibrosis and is involved in various kidney diseases (3). Oxidative stress caused by ROS mediates TGF- $\beta 1$-induced cellular responses in various cells $(4,5)$. Maintaining the redox balance and using antioxidants can delay the progression of renal interstitial fibrosis.

The therapeutic potential of Sirt1 activators has been explored in several diseases. Mammalian sirtuin 1 (Sirt1), a nicotinamide adenine dinucleotide $\left(\mathrm{NAD}^{+}\right)$-dependent protein class III deacetylase, is widely expressed throughout nearly all mammalian organs. Sirt1 is a key modulator of cell proliferation, hormone responses, stress responses, apoptosis and cell metabolism (6,7). A recent study demonstrated that Sirt1 activation attenuates renal fibrosis by inhibiting the TGF- $\beta 1 /$ Smad3 pathway (8). Sirt1 also interacts with TGF- $\beta 1$ signalling and inhibits apoptosis in TGF- $\beta 1$-treated mesangial cells (9). Moreover, a recent study highlighted the interaction of Sirt1 
with CTGF in cancer (10). However, it is unclear as to whether Sirtl activation exerts protective effects against UUO-induced tubulointerstitial fibrosis by suppressing renal oxidative stress and the TGF- $\beta 1 /$ CTGF signalling pathway.

Thus, in this study, we investigated the effects of the Sirt1 activator, SRT1720, a small molecule activator of Sirt1 that is more potent than resveratrol (11), on UUO-induced renal tubulointerstitial fibrosis and we aimed to determine whether these effects are mediated by the inhibition of oxidative stress and the TGF- $\beta 1 /$ CTGF pathway.

\section{Materials and methods}

Animal model and experimental design. Male 12-week-old CD-1 mice (Charles River Laboratories, Vital River, Beijing, China) were housed in the animal facilities at the Hebei Medical University with free access to food and water. UUO was created by the ligation of the left ureter with 4-0 silk through a left flank incision; during the procedure, the animals were under anesthesia induced with sodium pentobarbital. Sham-operated mice underwent the same procedure, except that the ureter was not ligated. The animals were randomly divided into 3 groups as follows: the sham-operated (sham) group, the group subjected to UUO (UUO) and the group subjected to UUO and treated with SRT1720 (SRT1720) (n=6/group). The Sirt1 activator, SRT1720 (100 mg/kg) (12) (Selleck Chemicals, Houston, TX, USA), was administered by intraperitoneal injection for 3 days prior to the operation and was continued for 7 days after UUO. The animals were sacrificed 7 days after UUO or sham operation, and the kidneys were harvested. All procedures were performed in compliance with the guidelines established by the Ethics Review Committee for Animal Experimentation of Hebei Medical University. This study was specifically approved by the Ethics Review Committee for Animal Experimentation of Hebei Medical University (Permit no. 2013-0666).

Histological analysis. The kidneys were fixed in $4 \%$ paraformaldehyde overnight and embedded in paraffin. Sections (2- $\mu \mathrm{m}$-thick) were stained with Masson's trichrome (Sigma, St. Louis, MO, USA). The fibrotic area was measured quantitatively using the HPIAS-2000 image analysis software (Champion Image Co., Wuhan, China). The results were expressed as the percentage area of interstitial fibrosis, as previously described (13).

Immunohistochemistry. The kidney sections (4- $\mu$ m-thick) were deparaffinised with xylene and rehydrated in graded ethanol. Endogenous horseradish peroxidase (HRP) activity was blocked with $3 \% \mathrm{H}_{2} \mathrm{O}_{2}$ in methanol for $30 \mathrm{~min}$. Antigen retrieval was performed by microwave treatment in $10 \mathrm{mM}$ citrate buffer for $15 \mathrm{~min}$. The sections were incubated with Sirt1 (sc-74465), $\alpha$-smooth muscle actin ( $\alpha$-SMA; sc-53015), tissue inhibitor of metalloproteinase (TIMP)-1 (sc-21734) and plasminogen activator inhibitor-1 (PAI-1; sc-8979) (Santa Cruz Biotechnology, Inc., Santa Cruz, CA, USA) antibodies overnight at $4^{\circ} \mathrm{C}$. The sections were then washed and incubated with biotinylated secondary antibody (sc-2364 and sc-2363; Santa Cruz Biotechnology, Inc.) and HRP-conjugated streptavidin. Labelling was visualised with 3,3-diaminobenzidine (DAB;
Sigma) to produce a brown colour, and the sections were counterstained with hematoxylin (Abcam, Cambridge, UK).

Detection of apoptotic cells. Apoptotic cells in the kidneys were identified using the Dead End ${ }^{\mathrm{TM}}$ Colorimetric terminal deoxynucleotidyltransferase-mediated dUTP nick-end labelling (TUNEL) system (Promega, Madison, WI, USA). Briefly, 4- $\mu \mathrm{m}$-thick paraffin-embedded sections were treated with freshly diluted $20 \mu \mathrm{g} / \mathrm{ml}$ proteinase $\mathrm{K}$ for $15 \mathrm{~min}$ at room temperature. Following treatment with equilibration buffer for $10 \mathrm{~min}$, the rTdT reaction mix was added for $60 \mathrm{~min}$ at $37^{\circ} \mathrm{C}$. Endogenous peroxidase activity was blocked by incubating the sections in $0.3 \% \mathrm{H}_{2} \mathrm{O}_{2}$ in phosphate-buffered saline (PBS) for 5 min. Streptavidin HRP solution was applied to each slide followed by incubation for $30 \mathrm{~min}$ at room temperature. The sections were developed with DAB. In randomly selected entire cortex fields, we captured 6 non-repeating micrographs (at x400 magnification) for each sample using a Leica DM2000 LED light microscope (Leica, Heidelberg, Germany). The number of TUNEL-positive apoptotic cells was counted per 100 tubular epithelial cells.

Western blot analysis. The whole kidneys were homogenised in ice-cold RIPA lysis buffer and centrifuged at $14,000 \mathrm{x}$ g for $25 \mathrm{~min}$ at $4^{\circ} \mathrm{C}$. The supernatants were collected. The protein concentration was measured by Bradford's method, and the supernatant was stored at $-80^{\circ} \mathrm{C}$. The cell lysates $(50 \mu \mathrm{g}$ of protein/lane) were loaded, separated by sodium dodecyl sulfate-polyacrylamide gel electrophoresis (SDS-PAGE), and transferred onto polyvinylidenedifluoride (PVDF) membranes (Millipore, Billerica, MA, USA). The membranes were incubated overnight at $4^{\circ} \mathrm{C}$ with the primary antibodies. Subsequently, the membranes were incubated with goat anti-rabbit IgG (sc-2004; Santa Cruz Biotechnology, Inc.) or goat anti-mouse IgG HRP conjugate (sc-2005; Santa Cruz Biotechnology, Inc.), and then immersed in ECL Plus Western Blotting detection reagent and exposed to Hyperfilm ECL (both from Amersham, Piscataway, NJ, USA). The intensity of the bands was measured using Lab Works 4.5 software (UVP, Upland, CA, USA). The primary antibodies were CTGF (ab6992; Abcam), TGF- $\beta 1$ (sc-130348), Sirt1 (sc-74465), collagen type I (Col1; sc-293182), $\alpha$-SMA (sc-53015), TIMP-1 (sc-21734), PAI-1 (sc-8979), E-Cadherin (E-cad; sc-71007), Bcl-2 (sc-56015), Bax (sc-20067) and $\beta$-actin (sc-130300) (Santa Cruz Biotechnology, Inc.).

Reverse transcription-quantitative PCR (RT-qPCR). Total RNA was isolated and CDNA was prepared from whole kidney samples using the SV Total RNA Isolation system and RT-PCR kits (both from Promega, Madison, WI, USA). Quantitative PCR (qPCR) was performed using SYBR Premix Ex Taq ${ }^{\mathrm{TM}}$ II. Real-time PCR reactions were performed on the Agilent Mx3000P QPCR system (Agilent, Santa Clara, CA, USA). The mRNA levels were normalised to the eukaryotic 18s rRNA expression level and were calculated using the $2(-\Delta \Delta \mathrm{Cq})$ method (14). The cycling conditions were as follows: initial denaturation at $95^{\circ} \mathrm{C}$ for $30 \mathrm{sec}$, followed by 40 cycles at $95^{\circ} \mathrm{C}$ for $5 \mathrm{sec}, 60^{\circ} \mathrm{C}$ for $30 \mathrm{sec}$ and $72^{\circ} \mathrm{C}$ for $30 \mathrm{sec}$. The primer sequences for Col1, $\alpha$-SMA, TIMP-1, PAI-1, E-cad, Bcl-2, Bax, CTGF, TGF- $\beta 1$, Sirt1 and 18S rRNA are shown in Table I. 
Table I. Primer sequences.

\begin{tabular}{|c|c|}
\hline Primer & Sequence \\
\hline Col1 & $\begin{array}{l}\text { F: 5'-CGCCATCAAGGTCTACTGC-3' } \\
\text { R: 5'-GAATCCATCGGTCATGCTCT-3' }\end{array}$ \\
\hline$\alpha-\mathrm{SMA}$ & $\begin{array}{l}\text { F: 5'-GAAGAGCATCCGACACTG-3' } \\
\text { R: 5'-GGCATAGCCCTCATAGATAG-3' }\end{array}$ \\
\hline TIMP-1 & $\begin{array}{l}\text { F: 5'-TGGGAAATGCCGCAGATA-3' } \\
\text { R: 5'-GCCAGGGAACCAAGAAGC-3' }\end{array}$ \\
\hline PAI-1 & $\begin{array}{l}\text { F: 5'-AAAAGTGTTTCAGCAGGTGGTC-3 } \\
\text { R: 5'-ACAAAGATGGCATCCGCAGT-3' }\end{array}$ \\
\hline E-cad & $\begin{array}{l}\text { F: 5'-CGACCCTGCCTCTGAATC-3' } \\
\text { R: 5'-AGCCAGCCTGTCTCCCTCT-3' }\end{array}$ \\
\hline $\mathrm{Bcl}-2$ & $\begin{array}{l}\text { F: 5'-GCTACCGTCGTGACTTCGC-3' } \\
\text { R: 5'-TCCCAGCCTCCGTTATCC-3' }\end{array}$ \\
\hline Bax & $\begin{array}{l}\text { F: 5'-CCAGGATGCGTCCACCAA-3' } \\
\text { R: 5'-AAGTAGAAGAGGGCAACCAC-3' }\end{array}$ \\
\hline CTGF & $\begin{array}{l}\text { F: 5'-GCCCAGACCCAACTATGATGC-3' } \\
\text { R: 5'-GCCCTTCTTAATGTTTTCCTCC-3' }\end{array}$ \\
\hline TGF- $\beta 1$ & $\begin{array}{l}\text { F: 5'-GGCGGTGCTCGCTTTGTA-3' } \\
\text { R: 5'-TTTCTCATAGATGGCGTTGTT-3' }\end{array}$ \\
\hline Sirt1 & $\begin{array}{l}\text { F: 5'-TAGGGAACCTTTGCCTCATCT-3' } \\
\text { R: 5'-CATTGTTGTTTGTTGCTTGGTC-3' }\end{array}$ \\
\hline 18sRNA & $\begin{array}{l}\text { F: 5'-AAACGGCTACCACATCCAAGG-3' } \\
\text { R: 5'-CCGCTCCCAAGATCCAACTAC-3' }\end{array}$ \\
\hline
\end{tabular}

Col1, collagen $1 ; \alpha$-SMA, $\alpha$-smooth muscle actin; TIMP-1, tissue inhibitor of metalloproteinase; PAI-1, plasminogen activator inhibitor-1; E-cad, E-cadherin; CTGF, connective tissue growth factor; TGF- $\beta 1$, transforming growth factor- $\beta 1$; Sirt 1 , sirtuin 1 .

Sirt1 deacetylase activity assay. For the measurement of Sirt1 deacetylase activity, the nuclear proteins were extracted and Sirt1 deacetylase activity was colourimetrically measured using the Sirt1 Deacetylase Activity assay kit (Genmed Scientifics Inc., Arlington, MA, USA) according to the manufacturer's instructions). The kit provides a Sirt1 substrate with an acetylated peptide fragment derived from p53, which is known to be deacetylated by Sirt1, that was pre-labeled by p-Nitroaniline. Following deacetylation by Sirt1, the aminopeptidase cleaved the deacetylated substrate and generated a highly chemiluminescent molecule group of p-Nitroaniline. The optical densities (OD) at a wavelength of $405 \mathrm{~nm}$ were recorded using a spectrophotometer (Diareader ELX800G; Dialab GmbH, Vienna, Austria).

Measurement of SOD, MDA, GSH and GPx levels. The superoxidedismutase (SOD; A001-1), malonaldehyde (MDA; A003-1), glutathione (GSH; A006-2) and glutathione peroxidase (GPx; A005) kits were purchased from Jiancheng Bioengineering Institute (Nanjing, China). First, the prepared fresh kidney tissue samples were homogenised in a saline solution to produce $10 \%$ kidney tissue homogenates. The homogenates were centrifuged at 3,500 rpm for $20 \mathrm{~min}$. The supernatant was collected to measure the levels of SOD (hydroxylamine method), MDA (thiobarbituric acid method), GSH (microplate assay) and GPx (colorimetric method) using the specific kits according to the manufacturer's instructions.

Statistical analysis. The data are presented as the means \pm SD. Statistical analysis was performed by one-way analysis of variance (ANOVA) using SPSS 11.0 statistical software. Significance was defined at a value of $\mathrm{P}<0.05$.

\section{Results}

Sirtl activation attenuates UUO-induced fibrosis. The Sirt1 protein (Fig. 1A-C), mRNA (Fig. 1D) and activity levels (Fig. 1E) were markedly decreased after UUO compared with the sham group. Treatment with the Sirt1 activator, SRT1720, increased the Sirt1 mRNA, protein and activity levels compared with the UUO group (Fig. 1). The percentage area of interstitial fibrosis in the kidneys from the sham-operated mice exhibited very little (if any) fibrosis in the tubules or interstitium. The percentage fibrotic area in the kidneys from the mice subjected to UUO was much higher than that of the sham group (Fig. 2A and B). Masson's trichrome staining revealed increased collagen deposition in the kidneys from the mice subjected to UUO compared with the sham-operated mice (Fig. 2A). We also analysed the total kidney tissue lysates by western blot analysis for Coll. An increase in Coll protein expression was observed in the obstructed kidneys compared with the sham group (Fig. 2D and E). Consistent with the protein expression of Col1, the mRNA level of Coll was increased in the mice subjected to UUO (Fig. 2C). SRT1720 substantially reversed these changes (Fig. 2).

A variety of cytokines are known to affect the process of interstitial fibrosis, such as PAI-1 and TIMP-1 (15). Tubular EMT occurs when losing epithelial markers, such as E-cad, and acquiring mesenchymal ones, such as $\alpha$-SMA (16). In this study, immunohistochemistry (Fig. 3) indicated that positive staining for PAI-1, TIMP-1 and $\alpha$-SMA was mainly found in the tubular epithelial cells in the kidneys from the mice subjected to UUO. Moreover, our results demonstrated that the mRNA and protein levels of PAI-1, TIMP-1 and $\alpha$-SMA all increased significantly in the obstructed kidneys of the mice in the UUO group compared to those of the sham group, as shown by western blot analysis and RT-qPCR (Fig. 2C-E). UUO led to decreased E-cad mRNA and protein expression. SRT1720 notably decreased the expression of $\alpha$-SMA, PAI- 1 and TIMP-1, and increased E-cad expression in the mice subjected to UUO (Fig. 2C-E).

Sirtl activation attenuates UUO-induced apoptosis in the kidneys of mice with UUO-induced fibrosis. Fig. 4A and B present the number of TUNEL-positive tubular epithelial cells in the mice subjected to UUO and treated with or without SRT1720. UUO significantly increased tubular epithelial cell apoptosis, which was markedly decreased by the delivery of SRT1720, compared with the sham-operated mice. Furthermore, in the obstructed kidneys from the mice in the UUO group, 7 days of UUO resulted in a significant decrease in 


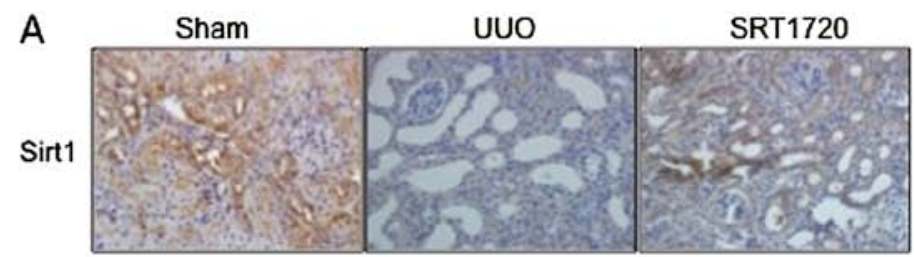

B

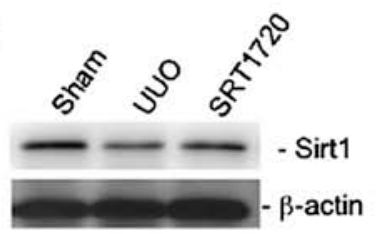

C

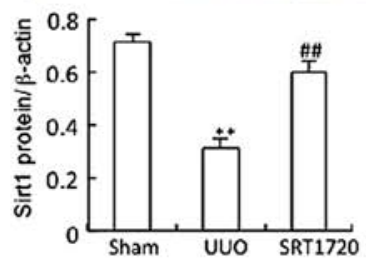

D

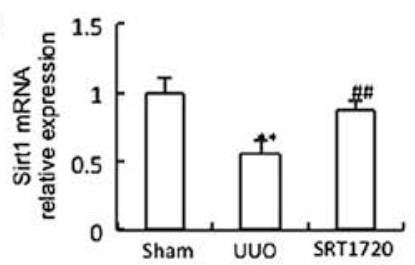

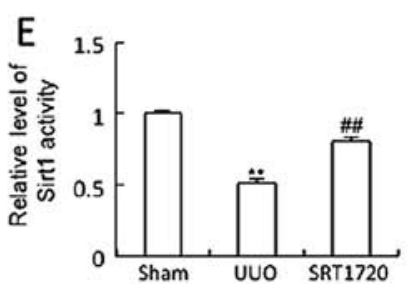

Figure 1. Effect of SRT1720 on unilateral ureteral obstruction (UUO)-induced sirtuin 1 (Sirt1) reduction. (A) Immunohistochemistry demonstrated that the positive staining for Sirt1 was primarily localised in the cytoplasm of renal tubular cells and was rarely found in glomeruli in the kidneys of the sham group. Sirt1 expression was markedly decreased in the kidneys in the UUO group. SRT1720 ameliorated the decrease in Sirt1 expression. Sirt1 protein expression (B and C), mRNA expression (D) and activity (E) all significantly decreased following UUO compared with to the sham group, and SRT1720 altered these reductions. Values are expressed as thye means $\pm \mathrm{SD}$. ${ }^{* *} \mathrm{P}<0.01$ vs. sham group; ${ }^{\# \#} \mathrm{P}<0.01$ vs. $\mathrm{UUO}$ group $(\mathrm{n}=6)$.
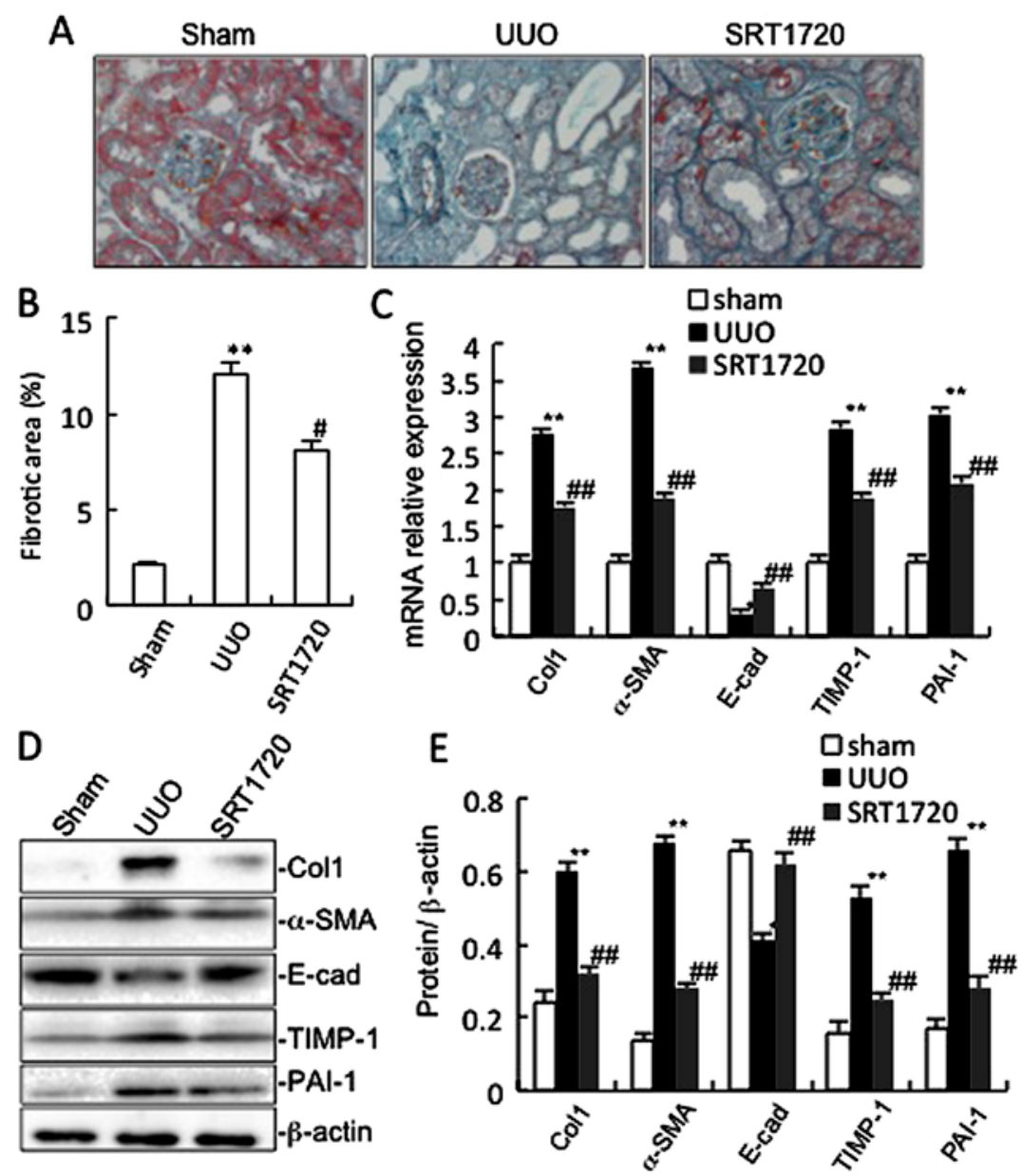

Figure 2. Effect of SRT1720 on unilateral ureteral obstruction (UUO)-induced fibrosis. (A) Masson's trichrome staining revealed increased collagen deposition in the kidneys from the mice subjected to UUO compared with the sham-operated mice. SRT1720 notably reduced the deposition in the kidneys in the mice in the UUO group. (B) The percentage areas of interstitial fibrosis in the sham, UUO and SRT1720 groups. (C and D) SRT1720 significantly attenuated the increase in Col1, $\alpha$-SMA, PAI-1, TIMP-1 expression, and the decrease in E-cad expression induced by UUO as shown by western blot analysis. (E) The increase in the mRNA expression levels of Col1, $\alpha$-SMA, PAI-1, TIMP-1 in the UUO group was attenuated by SRT1720 as shown by RT-qPCR. Values are expressed as means \pm SD. ${ }^{* *} \mathrm{P}<0.01$ vs. sham group; ${ }^{\# \#} \mathrm{P}<0.01$ vs. $\mathrm{UUO}$ group; ${ }^{~} \mathrm{P}<0.05$ vs. $\mathrm{UUO}(\mathrm{n}=6)$.

the protein and mRNA expression of Bcl-2, and in an increase in Bax expression compared with the sham-operated group.
SRT1720 administration attenuated the changes in Bcl-2 and Bax expression (Fig. 4C-E). 


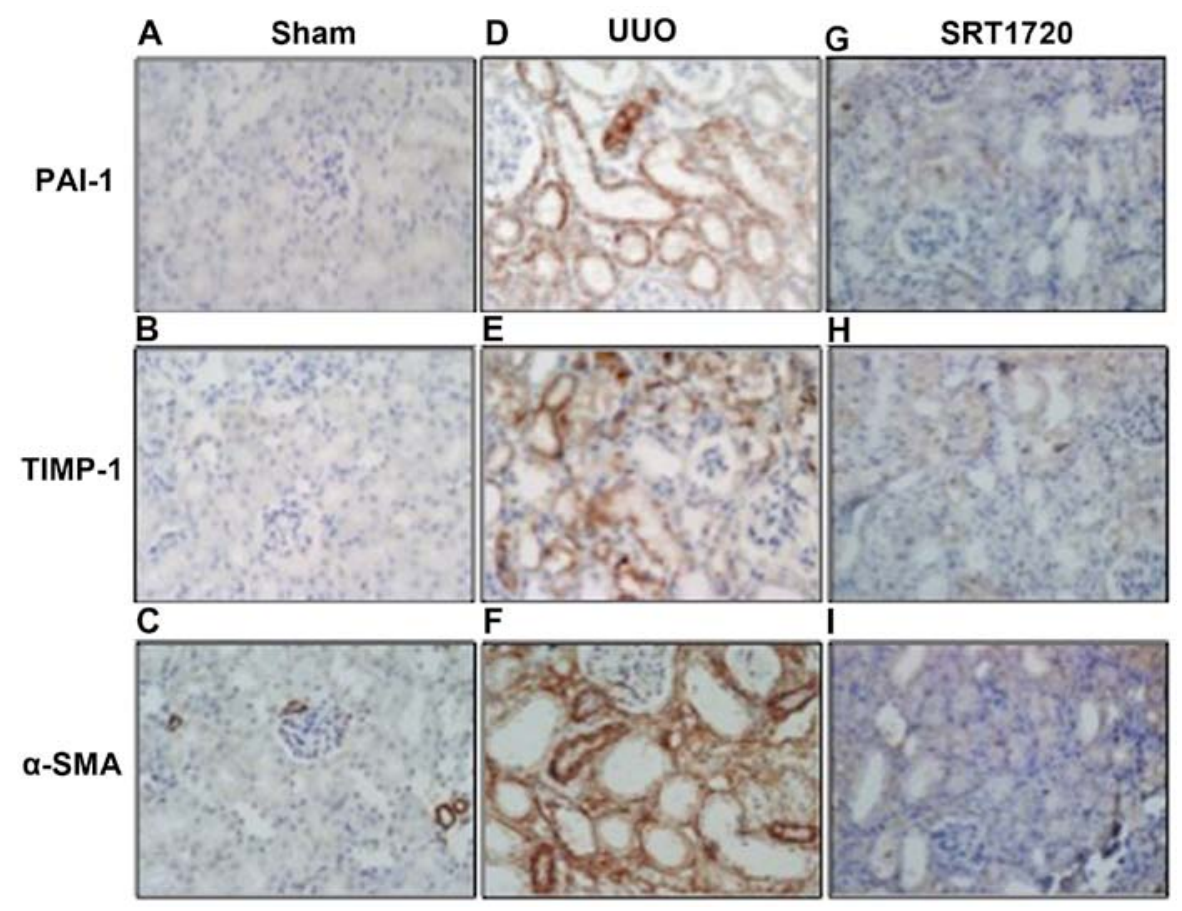

Figure 3. Delivery of SRT1720 downregulates the protein expression of PAI-1, TIMP-1 and $\alpha$-SMA in the kidneys of mice subjected to unilateral ureteral obstruction (UUO) as shown by immunohistochemistry. (A-C) Sham group. (D-F) Immunohistochemistry indicated that the positive staining was mainly found in the tubular epithelial cells in the UUO group. (G-I) SRT1720 significantly attenuated the expression in the kidneys of mice subjected to UUO.

A

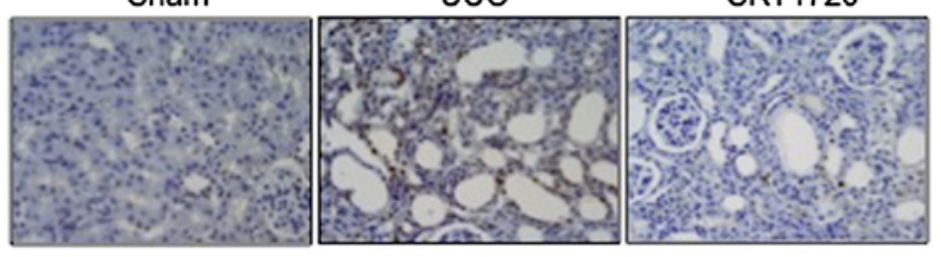

B

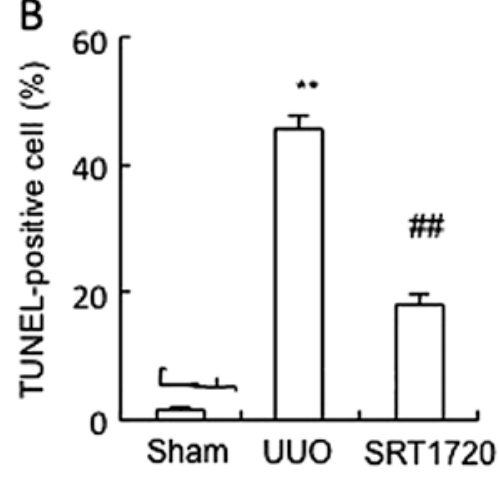

D

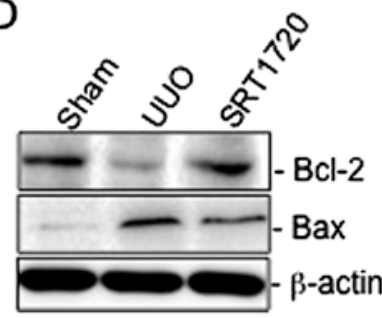

C 5 . 3 sham
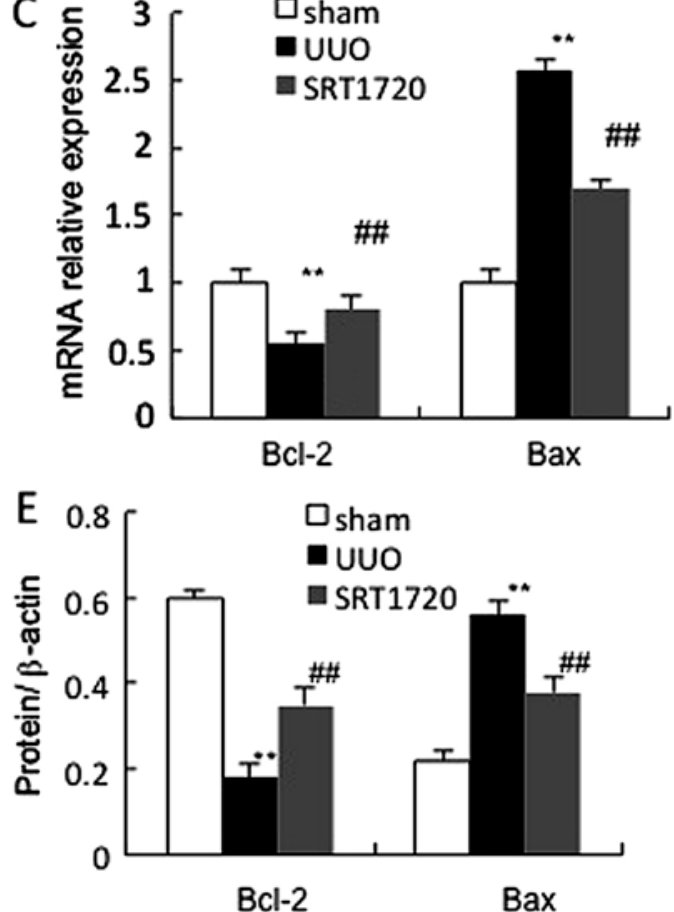

Figure 4. Treatment with SRT1720 attenuates unilateral ureteral obstruction (UUO)-induced apoptosis. (A) TUNEL assay indicated that the delivery of SRT1720 decreased the number of tubular epithelial cells containing positive nuclei compared to the kidneys from mice subjected to UUO. (B) The percentage of TUNELpositive cells in different groups. RT-qPCR (C) and western blot analysis (D and E) revealed a significant decrease in the mRNA and protein expression of Bcl-2, and an increase in Bax expression compared with the sham-operated group. SRT1720 attenuated the changes in Bcl-2 and Bax expression. Values are expressed as the means $\pm \mathrm{SD} .{ }^{* *} \mathrm{P}<0.01$ vs. sham group; ${ }^{\# \#} \mathrm{P}<0.01$ vs. UUO group $(\mathrm{n}=6)$. 
Table II. The results of oxidative stress analysis in sham and experimental groups ${ }^{\mathrm{a}}$.

\begin{tabular}{llrrr}
\hline Parameters & \multicolumn{1}{c}{ Unit } & sham & UUO & \multicolumn{1}{c}{ SRT1720 } \\
\hline MDA & nmol/mg protein & $7.12 \pm 1.06$ & $12.01 \pm 1.94^{\mathrm{b}}$ & $8.01 \pm 1.85^{\mathrm{c}}$ \\
SOD & U/mg protein & $15.78 \pm 1.25$ & $10.34 \pm 0.96^{\mathrm{b}}$ & $14.36 \pm 1.44^{\mathrm{c}}$ \\
GSH & $\mu$ mol/mg protein & $5.44 \pm 1.01$ & $4.89 \pm 1.20^{\mathrm{d}}$ & $8.62 \pm 0.87^{\mathrm{c}}$ \\
GPx & mU/mg protein & $378.34 \pm 28.25$ & $257.75 \pm 40.66^{\mathrm{b}}$ & $409.30 \pm 41.74^{\mathrm{c}}$ \\
\hline
\end{tabular}

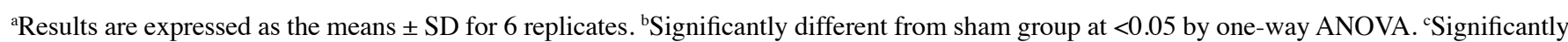
different from UUO group at $<0.05$ by one-way ANOVA. ${ }^{\mathrm{N}}$ No significantly difference from sham group at $>0.05$ by one-way ANOVA. UUO, unilateral ureteral obstruction; MDA, malondialdehyde; SOD, superoxide dismutase; GSH, reduced glutathione; GPx, glutathione peroxidase.
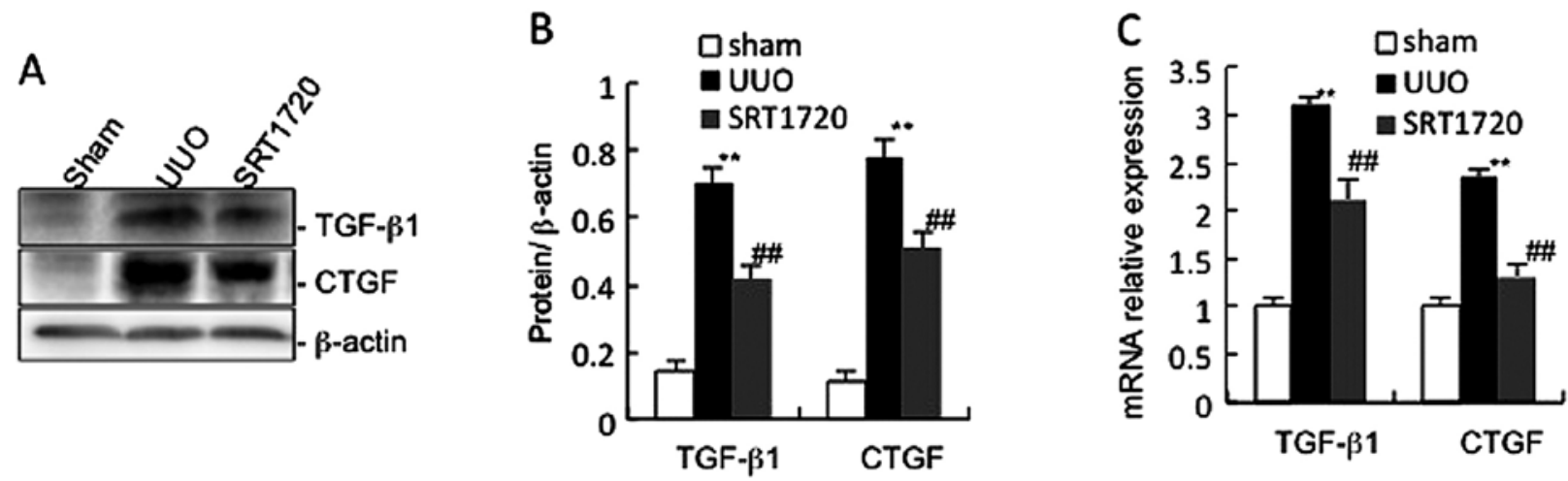

Figure 5. Treatment with SRT1720 inhibits the unilateral ureteral obstruction (UUO)-induced activation of the transforming growth factor- $\beta 1$ (TGF- $\beta 1$ )/ connective tissue growth factor (CTGF) pathway. SRT1720 decreased the UUO-induced increase in TGF- $\beta 1 / \mathrm{CTGF}$ protein and mRNA levels. Western blot analysis (A and B) and RT-qPCR (C). Values are expressed as the means \pm SD. ${ }^{* *} \mathrm{P}<0.01$ vs. sham group; ${ }^{\# *} \mathrm{P}<0.01$ vs. $\mathrm{UUO}$ group (n=6).

Sirtl activation inhibits the TGF- $\beta 1 / C T G F$ signalling pathway. It is well known that TGF- $\beta 1$ plays an important role in the physiology and pathology of kidney fibrosis (2). In this study, the TGF- $\beta 1$ level in the mice subjected to UUO was increased compared with the sham group. SRT1720 attenuated the UUO-induced increase in TGF- $\beta 1$ expression (Fig. 5). We then evaluated the TGF- $\beta 1$ downstream signalling molecule, CTGF. SRT1720 treatment also significantly blunted the increase in CTGF expression induced by UUO in mice.

Sirtl activation inhibits oxidative stress in kidneys. Oxidative stress has been postulated as an important contributing factor to fibrosis. Antioxidant enzymes, including SOD and GPx, protect against oxidative stress by converting free radicals or reactive oxygen intermediates to non-radical products. GSH is the major intracellular thiol that plays a critical role in the cellular defense against oxidative stress. It is clearly evident from Table II that UUO led to decreased SOD and GPx activity in the kidneys compared to the sham group. No significant change in the GSH level occurred after UUO. However, SRT1720 enhanced the renal SOD, GPx and GSH levels compared to the sham group. The increased activity of SOD, GPx and GSH in this study reflects a compensatory mechanism to the increased oxidative stress. With respect to the pro-oxidative biomarker, MDA (a major product of lipid peroxidation), the renal levels were significantly higher in the UUO group compared to the sham group, and the MDA levels decreased in the SRT1720 group compared with the UUO group (Table II).

\section{Discussion}

Renal fibrosis, whether the origin is inflammatory or immunological, obstructive, metabolic or systemic, inevitably progresses to end-stage renal disease with a progressive, irreversible decline in renal function. Therefore, the regulation of the renal fibrotic process provides new insight into the treatment and prevention of chronic kidney disease. The present study demonstrated that Sirtl deficiency accentuated renal tubular cell apoptosis and tubulointerstitial fibrosis. Conversely, the Sirtl activator, SRT1720, promoted the resistance of renal cells to oxidative stress during UUO. One of the primary mechanisms by which SRT1720 mediates its protective effects in the kidneys was by inhibiting TGF- $\beta 1 /$ CTGF expression.

Growing evidence indicates that sirtuins play a regulatory role in the process of different renal diseases. The antifibrotic function of Sirt1 has been reported in heart failure, cardiac fibrosis, hepatic steatosis, diabetic nephropathy and UUO (17). Furthermore, it has been demonstrated that Sirt1 is associated with TGF- $\beta$ in endothelial, vascular smooth muscle and bronchial epithelial cells. The overexpression of Sirt1 attenuates TGF- $\beta 1$-induced extracellular matrix expression in cultured mesangial cells (8). In this study, we observed that in the UUO-obstructed mouse kidneys, Sirt1 expression and activity both markedly decreased. The the Sirt1 activator, SRT1720, was administered. Our results demonstrated that SRT1720 increased the Sirt1 levels and activity, and attenuated the renal upregulation of matrix accumulation and epithelial- 
mesenchymal transition. We also demonstrated that SRT1720 also effectively attenuated apoptosis induced by UUO. The resutls of TUNEL assay also indicated that UUO increased the apoptotic rate, which was effectively inhibited by treatment with SRT1720. The results of western blot analysis and RT-qPCR revealed that Sirt1 overexpression increased Bcl-2 protein and mRNA expression, and decreased the protein and mRNA levels of Bax. The protective effects of Sirt1 on apoptosis have been reported in several studies in multiple organs and tissues, against injury from diverse causes. $\mathrm{H}_{2} \mathrm{O}_{2}$-induced apoptosis has been shown to be significantly attenuated in Sirt1-overexpressing murine mesangial cells, but to be enhanced with Sirt1-knockdown in MMCs (18). The downregulation of Sirt1 expression in diabetes promotes podocyte apoptosis (19). In another study, the UUO model of kidney injury markedly induced more renal apoptosis and fibrosis in Sirt1 ${ }^{+/-}$mice compared to the wild-type controls, and the pharmacological Sirt1 activation substantially attenuated apoptosis and fibrosis in wild-type mice (17). These data further indicate that Sirt1 may be a promising therapeutic target as a common pathway in renal apoptosis and fibrosis.

The pathogenesis of renal fibrosis has been depicted as a continuum of 4 overlapping phases: priming (localised inflammatory response), activation (activation and recruitment of matrix producing cells), execution (accumulation of matrix protein) and progression (loss of cells and renal function) (20). TGF- $\beta 1$ and CTGF, key mediators of kidney fibrosis, were identified based on their contribution to these phases (2). TGF- $\beta 1$, an ubiquitously expressed cytokine, also promotes tissue repair, but its excessive action can lead to diverse profibrotic events in the liver, lung, kidney and breast (21). CTGF has many diverse functions, such as angiogenesis, fibrosis and apoptosis (22). In this study, we found that the expression of CTGF and TGF- $\beta 1$ increased in the UUO-obstructed kidneys, which was accompanied by a significant upregulation of matrix accumulation, EMT and apoptosis. This result is consistent with the observation that the expression of TGF- $\beta 1$ and CTGF is increased in human and experimental models of chronic kidney disease (correlating with the severity of renal fibrosis) (23-25).

Oxidative stress results from an imbalance between ROS production and elimination. Modi et al demonstrated that the MDA levels were significantly higher in rats with ureteral obstruction compared with the sham-treated group (26). In response to ROS, antioxidant enzymes, including SOD, are thought to protect cellular function. A deficiency in these antioxidant enzymes enhances ROS production and renal tubulointerstitial fibrosis (27). Our results demonstrated that under normal conditions, kidney cells contain low levels of the pro-oxidative biomarker, MDA. Abundant antioxidant enzymes (such as SOD and GPx) are likely to play an important role in protecting the kidneys against oxidative stress. However, UUO led to decreased SOD and GPx activity compared to the normal group. There was no significant difference in the GSH levels. The MDA levels were markedly higher than the normal group.

In the present study, to the best of our knowledge, we demonstrated for the first time that SRT1720 treatment decreased TGF- $\beta 1$ production and CTGF activation in kidney tissues obtained from mice subjected to UUO. In addition,
SRT1720 treatment reversed the oxidative stress induced by UUO.

Taken together, these data suggest that the Sirtl activator, SRT1720, exerts protective effects against UUO-induced tubulointerstitial fibrosis, in part through the suppression of renal oxidative stress and the TGF- $\beta 1 / \mathrm{CTGF}$ signalling pathway. Targeting Sirt1 with pharmacological activators may thus improve renal function during conditions that induce tubulointerstitial fibrosis, including ureteral obstruction.

\section{Acknowledgements}

This study was supported by grants from the National Natural Science Foundation of China (no. 81200532) and the preferred fund of Hebei Province Postdoctoral Scientific Research Projects (no. B2015003028).

\section{References}

1. Klahr S and Morrissey J: Obstructive nephropathy and renal fibrosis. Am J Physiol Renal Physiol 283: F861-F875, 2002.

2. Leask A and Abraham DJ: TGF-beta signaling and the fibrotic response. FASEB J 18: 816-827, 2004.

3. Devarajan P: Update on mechanisms of ischemic acute kidney injury. J Am Soc Nephrol 17: 1503-1520, 2006.

4. Chiu C, Maddock DA, Zhang Q, Souza KP, Townsend AR and Wan Y: TGF-beta-induced p38 activation is mediated by Rac1-regulated generation of reactive oxygen species in cultured human keratinocytes. Int J Mol Med 8: 251-255, 2001.

5. Herrera B, Alvarez AM, Sánchez A, Fernández M, Roncero C, Benito M and Fabregat I: Reactive oxygen species (ROS) mediates the mitochondrial-dependent apoptosis induced by transforming growth factor $(\beta)$ in fetal hepatocytes. FASEB J 15: 741-751, 2001.

6. Michan S and Sinclair D: Sirtuins in mammals: Insights into their biological function. Biochem J 404: 1-13, 2007.

7. Kim DH, Jung YJ, Lee JE, Lee AS, Kang KP, Lee S, Park SK, Han MK, Lee SY, Ramkumar KM, et al: SIRT1 activation by resveratrol ameliorates cisplatin-induced renal injury through deacetylation of p53. Am J Physiol Renal Physiol 301: F427-F435, 2011.

8. Huang XZ, Wen D, Zhang M, Xie Q, Ma L, Guan Y, Ren Y, Chen J and Hao CM: Sirtl activation ameliorates renal fibrosis by inhibiting the TGF- $\beta /$ Smad3 pathway. J Cell Biochem 115: 996-1005, 2014.

9. Kume S, Haneda M, Kanasaki K, Sugimoto T, Araki S, Isshiki K, Isono M, Uzu T, Guarente L, Kashiwagi A, et al: SIRT1 inhibits transforming growth factor beta-induced apoptosis in glomerular mesangial cells via Smad7 deacetylation. J Biol Chem 282: 151-158, 2007.

10. Mao B, Hu F, Cheng J, Wang P, Xu M, Yuan F, Meng S, Wang Y, Yuan $Z$ and $B i$ W: SIRT1 regulates YAP2-mediated cell proliferation and chemoresistance in hepatocellular carcinoma. Oncogene 33: 1468-1474, 2014.

11. Milne JC, Lambert PD, Schenk S, Carney DP, Smith JJ, Gagne DJ, Jin L, Boss O, Perni RB, Vu CB, et al: Small molecule activators of SIRT1 as therapeutics for the treatment of type 2 diabetes. Nature 450: 712-716, 2007.

12. Gano LB, Donato AJ, Pasha HM, Hearon CM Jr, Sindler AL and Seals DR: The SIRT1 activator SRT1720 reverses vascular endothelial dysfunction, excessive superoxide production, and inflammation with aging in mice. Am J Physiol Heart Circ Physiol 307: H1754-H1763, 2014.

13. Shimizu H, Maruyama S, Yuzawa Y, Kato T, Miki Y, Suzuki S, Sato W, Morita Y, Maruyama H, Egashira K, et al: Antimonocyte chemoattractant protein-1 gene therapy attenuated renal injury induced by protein-overload proteinuria. J Am Soc Nephrol 14: 1496-1505, 2003.

14. Livak KJ and Schmittgen TD: Analysis of relative gene expression data using real-time quantitative PCR and the 2(- $\Delta \Delta \mathrm{C}(\mathrm{T}))$ method. Methods 25: 402-408, 2001.

15. Duymelinck C, Deng JT, Dauwe SE, De Broe ME and Verpooten GA: Inhibition of the matrix metalloproteinase system in a rat model of chronic cyclosporine nephropathy. Kidney Int 54: $804-818,1998$ 
16. Yang J and Liu Y: Dissection of key events in tubular epithelial to myofibroblast transition and its implications in renal interstitial fibrosis. Am J Pathol 159: 1465-1475, 2001.

17. He W, Wang Y, Zhang MZ, You L, Davis LS, Fan H, Yang HC, Fogo AB, Zent R, Harris RC, et al: Sirtl activation protects the mouse renal medulla from oxidative injury. J Clin Invest 120: 1056-1068, 2010.

18. Kume S, Haneda M, Kanasaki K, Sugimoto T, Araki S, Isono M, Isshiki K, Uzu T, Kashiwagi A and Koya D: Silent information regulator 2 (SIRT1) attenuates oxidative stress-induced mesangial cell apoptosis via p53 deacetylation. Free Radic Biol Med 40: 2175-2182, 2006.

19. Chuang PY, Dai Y, Liu R, He H, Kretzler M, Jim B, Cohen CD and He JC: Alteration of forkhead box $\mathrm{O}$ (foxo4) acetylation mediates apoptosis of podocytes in diabetes mellitus. PLoS One 6: e23566, 2011.

20. Liu Y: Cellular and molecular mechanisms of renal fibrosis. Nat Rev Nephrol 7: 684-696, 2011.

21. Franklin TJ: Therapeutic approaches to organ fibrosis. Int J Biochem Cell Biol 29: 79-89, 1997.

22. Sánchez-López E, Rodrigues Díez R, Rodríguez Vita J, Rayego Mateos S, Rodrigues Díez RR, Rodríguez García E, Lavoz Barria C, Mezzano S, Egido J, Ortiz A, Ruiz-Ortega M and Selgas R: Connective tissue growth factor (CTGF): a key factor in the onset and progression of kidney damage. Nefrologia 29: 382-391, 2009 (In Spanish).
23. Border WA and Noble NA: Transforming growth factor beta in tissue fibrosis. N Engl J Med 331: 1286-1292, 1994.

24. Ito Y, Aten J, Bende RJ, Oemar BS, Rabelink TJ, Weening JJ and Goldschmeding R: Expression of connective tissue growth factor in human renal fibrosis. Kidney Int 53: 853-861, 1998.

25. Yokoi H, Sugawara A, Mukoyama M, Mori K, Makino H, Suganami T, Nagae T, Yahata K, Fujinaga Y, Tanaka I, et al: Role of connective tissue growth factor in profibrotic action of transforming growth factor-beta: A potential target for preventing renal fibrosis. Am J Kidney Dis 38 (Suppl 1): S134-S138, 2001.

26. Modi KS, Morrissey J, Shah SV, Schreiner GF and Klahr S: Effects of probucol on renal function in rats with bilateral ureteral obstruction. Kidney Int 38: 843-850, 1990.

27. Sunami R, Sugiyama H, Wang DH, Kobayashi M, Maeshima Y, Yamasaki Y, Masuoka N, Ogawa N, Kira S and Makino H: Acatalasemia sensitizes renal tubular epithelial cells to apoptosis and exacerbates renal fibrosis after unilateral ureteral obstruction. Am J Physiol Renal Physiol 286: F1030-F1038, 2004. 